\title{
Poor Mobility in Hospitalized Adults of All Ages
}

\author{
Alison M. Mudge, PhD ${ }^{1,2 *}$, Prue McRae, MPhil2,3, Kirstie McHugh, MPhty ${ }^{4}$, Lauren Griffin, MPhty ${ }^{4}$, Andrew Hitchen, MPhty ${ }^{4}$,
} James Walker, MPhty ${ }^{4}$, Mark Cruickshank, BPhty ${ }^{5}$, Norman R. Morris, PhD ${ }^{4,6}$, Suzanne Kuys, PhD ${ }^{4}$

IInternal Medicine and Aged Care, Royal Brisbane and Women's Hospital, Brisbane, Queensland, Australia; 2University of Queensland School of Medicine, Brisbane, Queensland, Australia; ${ }^{3}$ Safety and Quality Unit, Royal Brisbane and Women's Hospital, Brisbane, Queensland, Australia; ${ }^{4}$ School of Allied Health Sciences, Griffith University, Brisbane, Queensland, Australia; ${ }^{5}$ Physiotherapy Department, Royal Brisbane and Women's Hospital, Brisbane, Queensland, Australia; ${ }^{6}$ Griffith Health Institute, Brisbane, Queensland, Australia.

Low levels of activity in hospital inpatients contribute to functional decline. Previous studies have shown low levels of activity in older inpatients, but few have investigated younger inpatients (aged $<65$ years). This observational study measured activity in older (aged $\geq 65$ years) and younger hospital inpatients on 3 wards (medical, surgical, oncology) in a major teaching hospital in Brisbane, Australia, as part of a quality-improvement intervention to enhance mobility. Using structured behavioral mapping protocols, participants were observed for 2-minute intervals throughout 4, 4-hour daytime observation periods. The proportion of time spent at different activity levels was calcu- lated for each participant, and time spent standing, walking or wheeling was compared between age group and wards. There were 3272 observations collected on 132 participants (median, 30 per patient; range, 9-35). The most time was spent lying in bed (mean 57\%), with $9 \%$ standing or walking. There were significant differences among wards, but no difference between older and younger subgroups. Low mobility is common in adult inpatients of all ages. Behavioral mapping provided measures suitable for use in quality improvement. Journal of Hospital Medicine 2016;11:289291. (C) 2016 Society of Hospital Medicine
Low mobility is common in hospitalized older patients, and an independent predictor of poor functional outcomes. ${ }^{1-4}$ Few studies have included younger patients, but care models that support early mobility may reduce functional decline, enhance recovery, and reduce length of stay in older and mixed-age populations. ${ }^{5,6}$ Barriers to mobility are complex and include patient symptoms and tethers, health provider behavior, team communication, and leadership, device availability, and environmental factors. ${ }^{7-11}$ These contextual factors may differ even within a hospital between patient groups and ward settings. Simple measures to quantify mobility patterns would help address these barriers by providing opportunities for audit and feedback. Although accelerometry is the gold standard method for research, it requires equipment, analysis skills, and patient consent, which limits application in clinical practice. Behavioral mapping is a systematic method of observation developed in stroke patients, which is simple, objective, and requires no direct patient or staff participation, ${ }^{12}$ and physical activity levels estimated from behavioral mapping are similar to those identified by accelerometry. ${ }^{3,13,14}$ In the context of a phased quality-improvement project aiming to

\footnotetext{
*Address for correspondence and reprint requests: Alison M. Mudge, Internal Medicine and Aged Care, 3rd Floor, James Mayne Building, Royal Brisbane and Women's Hospital, Butterfield St. Herston 4029, Brisbane, Queensland, Australia; Telephone: 61736560854;

E-mail: alison.mudge@health.qld.gov.au

Additional Supporting Information may be found in the online version of this article.

Received: July 30, 2015; Revised: November 19, 2015; Accepted: December 4, 2015

2016 Society of Hospital Medicine DOI 10.1002/jhm.2536

Published online in Wiley Online Library (Wileyonlinelibrary.com).
}

reduce functional decline, ${ }^{15}$ we undertook a crosssectional audit of mobility on 3 different wards using behavioral mapping, and examined differences among wards and between older (aged 65 years or more) and younger patients.

\section{METHODS}

This prospective observational study used crosssectional sampling from a 26-bed general medical ward, a 30-bed oncology ward, and a 24-bed vascular surgical ward in a 900-bed tertiary teaching hospital in Brisbane, Australia. Sampling was undertaken during 4 observation periods (2 mornings [1000-1400] and 2 afternoons [1400-1800]) within 10 days in May 2013. All patients on each ward for each period were observed unless they were receiving end-of-life care. Structured observations were undertaken using behavioral mapping protocols similar to those previously described in stroke and general medical patients, ${ }^{12,13}$ with each patient room visited in the same sequence. Participants in each room were observed for a 2minute period (up to 4 participants could be observed concurrently in shared rooms) before moving to the next room, and the sequence was repeated in the same order for the whole 4-hour period, with a single 15-minute break. Depending on ward size and layout, this provided 12 to 17 observations per participant for each 4-hour period (each individual observed every 12-18 minutes). Observations were undertaken by 4 trained physiotherapy student observers using a predetermined set of mutually exclusive levels (lying in bed, sitting in or on the bed, sitting on a chair, standing, actively wheeling, or walking). The study was approved by the Royal Brisbane and Women's Hospital Human 


\begin{tabular}{|c|c|c|c|}
\hline & $\begin{array}{c}\text { All Ages, } \\
\mathrm{n}=132, \text { Median } \\
\text { Observations } 29.5, \\
\text { Range } 6-65^{\star}\end{array}$ & $\begin{array}{c}\text { Aged }<65 \text { Years, } \\
\mathrm{n}=61, \text { Median } \\
\text { Observations } 30 \\
\text { Range } 6-65\end{array}$ & $\begin{array}{c}\text { Aged } \geq 65 \text { Years, } \\
n=67, \text { Median } \\
\text { Observations } 27, \\
\text { Range 6-65 }\end{array}$ \\
\hline \multicolumn{4}{|l|}{ Location } \\
\hline Bedroom & 85.1 (13.3) & 84.6 (13.4) & $85.5(12.9)$ \\
\hline Bathroom & $3.0(4.0)$ & $2.6(3.9)$ & $3.4(4.1)$ \\
\hline Hall & $2.9(4.6)$ & $3.4(5.4)$ & $2.7(4.0)$ \\
\hline Lounge & $0.3(1.9)$ & 0 & $0.6(2.7)$ \\
\hline Off ward/other & $8.6(11.6)$ & $9.3(11.4)$ & $7.8(11.1)$ \\
\hline \multicolumn{4}{|l|}{ Physical activity } \\
\hline Lie in bed & $57.4(30.0)$ & $59.4(29.4)$ & $55.5(31.6)$ \\
\hline Sit on bed & $21.0(23.2)$ & $16.9(19.9)$ & $24.7(25.7)$ \\
\hline Sit on chair & $12.6(22.9)$ & $14.0(25.6)$ & $11.9(20.9)$ \\
\hline Stand/walk/wheel & $9.0(9.3)$ & $9.6(9.6)$ & $8.0(8.5)$ \\
\hline
\end{tabular}

Research Ethics Committee as part of a qualityimprovement activity, and individual consent was not required. No clinical data except age and gender were collected for participants. The nurse unit manager for each ward was introduced to the observers and aware that observations were being conducted.

Patients who were observed for less than one-half of an observation period were excluded so that all participants contributed at least 2 hours of observational data, up to a maximum of 16 hours. The number of valid observations for each participant (excluding time off ward or behind curtains if the level was not apparent) was calculated and used to derive the proportion of valid observations spent at each level for each participant. The proportion of observations at each level was summarized across all participants using frequency distributions and summary statistics. For ease of presentation, mean percentage of observed time in each activity was presented. However, as data were not normally distributed, statistical comparisons were undertaken using the Kruskal-Wallis test, comparing the distribution of time spent upright (standing, walking, or actively wheeling) between groups (age group and ward). Interaction between age and ward effects was sought using generalized linear modeling.

\section{RESULTS}

Valid observations (at least 2 hours in 1 or more observation period) were available for 132 patients (48 medical, 50 oncology, and 34 surgery). Of these, $67(51 \%)$ were aged $\geq 65$ years $(54 \%$ medical, $44 \%$ oncology, $56 \%$ surgery) and $62(47 \%)$ were male. There were a total of 3891 observations of location (median, 30 per patient; range, 9-65). Participants were observed in the bedded area $85.1 \%$ of observations, with $3.1 \%$ in the bathroom, $3.2 \%$ in the hallway or patient lounge, and $8.6 \%$ off ward. Allowing for time off ward and behind curtains, when observers
TABLE 2. Mean (Standard Deviation) Percentage of Observations for Each Behavior Compared by Ward Type

\begin{tabular}{lccc}
\hline & $\begin{array}{c}\text { Medical, } \\
\mathrm{n}=48, \text { Median } \\
\text { Observations 30, } \\
\text { Range 7-59 }\end{array}$ & $\begin{array}{c}\text { Oncology, } \\
\mathrm{n}=50, \text { Median } \\
\text { Observations 25, } \\
\text { Range 6-52 }\end{array}$ & $\begin{array}{c}\text { Surgical, } \\
\mathrm{n}=34, \text { Median } \\
\text { Observations 31, } \\
\text { Range 17-65 }\end{array}$ \\
\hline Location & & & \\
Bedroom & $89.1(11.4)$ & $81.3(13.6)$ & $85.3(14.1)$ \\
Bathroom & $2.8(4.1)$ & $3.1(3.8)$ & $3.1(4.2)$ \\
Hall & $1.5(2.5)$ & $5.3(6.1)$ & $1.5(2.7)$ \\
Lounge & $0.5(2.0)$ & $0.4(2.5)$ & 0 \\
Off ward/other & $6.2(10.2)$ & $10.0(11.9)$ & $10.1(12.6)$ \\
Physical activity & & & \\
Lie in bed & $53.3(31.4)$ & $56.1(30.2)$ & $65.1(27.0)$ \\
Sit on bed & $30.3(29.5)$ & $13.4(16.1)$ & $19.0(17.0)$ \\
Sit on chair & $8.2(14.7)$ & $19.1(29.1)$ & $9.3(20.4)$ \\
Stand/walk/wheel & $8.2(8.4)$ & $11.4(9.7)$ & $6.5(9.4)$ \\
\hline
\end{tabular}

could not be sure of their activity level, 3272 valid observations were available for physical activity.

More than half of the observed time (mean $57.4 \%$ ) was spent lying in bed, $33.6 \%$ sitting on the bed or chair, and $9.0 \%$ standing, walking, or wheeling. Across all observation periods, 39/132 (29.5\%) participants were never observed to be standing, walking or wheeling, and $7.6 \%$ were in bed at all observations. Comparing older and younger patients (Table 1), there was no difference in the time spent in active upright postures (median, $6.1 \%$ in older vs $7.4 \%$ in younger; $P=0.30$ ). Table 2 summarizes descriptive data for the different wards. In the medical and surgical wards, $84 \%$ of the time was spend in or on the bed, and only $16 \%$ of the time was spent sitting in a chair or in active upright postures. Surgical patients, in particular, spent twothirds of observation time lying flat in bed, whereas medical patients spent more time sitting up on the bed. On statistical testing, time spent standing/walking/ wheeling was significantly lower on the surgical ward (median, 4\%; interquartile range [IQR], 0-10 for surgery; median, 7\%; IQR, 0-13 for medical; and median, $10 \%$; IQR 3-17 for oncology; $P=0.015)$. This was also reflected in a higher proportion of surgical patients never seen in an active upright position $(44.1 \%$ compared to $27.1 \%$ medical and $22.0 \%$ oncology). Multivariate modeling showed no significant interaction between age and ward.

\section{DISCUSSION}

This observational cross-sectional study extends previous observations of hospital inpatients to include a wider variety of patient types and ages. Observing 132 patients on medical, surgical, and oncology wards for up to 16 hours of weekday time, we found that patients spent only $9 \%$ in active upright postures, with significantly lower mobility on the surgical ward but no significant differences between older and younger patients. 
Previous studies in older general medical patients using behavioral mapping ${ }^{13}$ or accelerometers ${ }^{2,3}$ have shown $71 \%$ to $83 \%$ of time spent in bed, and $4 \%$ spent standing or walking, similar to our findings, although methodological differences between studies (eg, patient selection and time windows) caution against direct comparison. We identified different levels of physical activity on the surgical, medical, and oncology wards. This may reflect differences in patient case-mix, ward environment, and/or ward culture. The medical and oncology wards each have a patient lounge, providing a potential walking destination, although only a small amount of patients' time was observed in these areas, suggesting that they may not fulfil their purpose. The oncology ward has a well developed "wellness" focus. The oncology and medical wards were actively involved in a qualityimprovement intervention to improve early patient mobility at the time of the audit, ${ }^{15}$ whereas the surgical ward was at the precommencement (information gathering) stage. The data collected within this audit have formed part of the feedback cycle for staff involved in the improvement intervention. Repeat measurement will be undertaken on the surgical ward to help evaluate the impact of the intervention, and serial measurement will be undertaken in future participating wards to investigate the responsiveness of this measurement method.

Although the literature has focused on poor mobility in hospitalized elders, we did not find any better mobility in younger patients, suggesting that barriers to mobility are not confined to the elderly. Whereas individualized mobility assessment and support may be more important in the elderly, ${ }^{16}$ addressing cultural and environmental issues such as promoting accountability for early ambulation, providing patients and families with permission and encouragement to ambulate, and ensuring accessible walking destinations may benefit patients of all ages.

Behavioral mapping has strengths and weaknesses compared to other methods such as accelerometry or patient/nurse report. Observations are conducted by an independent observer not involved in care and include all ward inpatients, providing a generalizable sample, as the observation protocol does not pose a participation burden for patient or ward staff. However, the cross-sectional nature may oversample longer-stay patients, the intermittent observation protocol tends to overestimate time spent upright, ${ }^{14}$ the labor-intensive nature of observations means choosing a limited time window (in our case 1000-1800), and the minimum time and observation frequency to generate reliable data remain uncertain. Further studies examining reliability, validity, and responsiveness would support the utility of this method for quality improvement.

In summary, this study shows that mobility is limited in older and younger adult inpatients across a range of inpatient wards, and that physical activity practices vary among wards. Interventions to enhance hospital mobility should include patients of all ages, and need to be tailored to local mobility practices, barriers, and enablers.

\section{Acknowledgements}

The authors thank the staff of wards 6AS, 9BN, and 7BW for participating in this project.

Disclosure: Nothing to report.

\section{References}

1. Brown C, Friedkin R, Inouye S. Prevalence and outcomes of low mobility in hospitalized older patients. J Am Geriatr Soc. 2004;52(8): 1263-1270.

2. Brown C, Redden D, Flood K, Allman R. The underrecognized epidemic of low mobility during hospitalization of older adults. J Am Geriatr Soc. 2009;57:1660-1665.

3. Pedersen M, Bodilsen A, Petersen J, et al. Twenty-four-hour mobility during acute hospitalization in older medical patients. J Gerontol A Biol Sci Med Sci. 2013;68(3):331-337.

4. Zisberg A, Shadmi E, Sinoff G, Gur-Yeish N, Srulovici E, Admi H. Low mobility during hospitalization and functional decline in older adults. I Am Geriatr Soc. 2011;59(2):266-273.

5. Mudge A, Giebel A, Cutler A. Exercising body and mind: an integrated approach to functional independence. J Am Geriatr Soc. 2008; 56:630-635.

6. Mundy L, Leet T, Darst K, Schnitzler M, Dunagen W. Early mobilization of patients hospitalized with community-acquired pneumonia. Chest. 2003;124(124):883-889.

7. Boltz M, Capezuti E, Shabbat N. Nursing staff perceptions of physical function in hospitalized older adults. App Nurs Res. 2011;24:215222.

8. Brown C, Williams B, Woodby L, Davis L, Allman R. Barriers to mobility during hospitalization from the perspectives of older patients and their nurses and physicians. J Hosp Med. 2007;2:305-313.

9. Doherty-King B, Bowers B. How nurses decide to ambulate hospitalized older adults: development of a conceptual model. Gerontologist. 2011;51(6):786-797.

10. Hoyer E, Brotman D, Chan K, Needham D. Barriers to early mobility of hospitalized general medicine patients. Survey development and validation. Am J Phys Med Rehabil. 2015;94:304-312.

11. So C, Pierlussi E. Attitudes and expectations regarding exercise in the hospital of hospitalized older adults: a qualitative study. J Am Geriatr Soc. 2012;60:713-718.

12. Bernhardt J, Dewey H, Thrift A, Donnan G. Inactive and alone. Physical activity within the first 14 days of acute stroke unit care. Stroke. 2004;35:1005-1009.

13. Kuys S, Dolecka U, Guard A. Activity level of hospital medical inpatients: an observational study. Arch Gerontol Geriatr. 2012;55:417421.

14. Kramer S, Cumming T, Churilov L, Bernhardt J. Measuring activity levels at an acute stroke ward: comparing observations to a device. Biomed Res Int. 2013;2013:460482.

15. Mudge A, McRae P, Cruickshank M. Eat walk engage: an interdisciplinary collaborative model to improve care of hospitalized elders. Am J Med Qual. 2015;30(1):5-13.

16. Covinsky K, Pierlussi E, Johnston C. Hospitalization-associated disability. "She was probably able to ambulate, but I'm not sure". JAMA. 2011;306(16):1782-1793. 